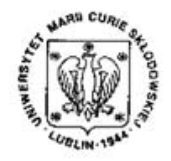

Annales UMCS

Informatica

Lublin-Polonia

Sectio AI

http://www.annales.umcs.lublin.pl/

\title{
Difference schemes of arbitrary order of accuracy for semilinear parabolic equations
}

\author{
Magdalena Lapinska-Chrzczonowicz* \\ Institute of Mathematics and Computer Science, \\ The John Paul II Catholic University of Lublin, \\ Al. Racławickie 14, 20-950 Lublin, Poland.
}

\begin{abstract}
The Cauchy problem for a semilinear parabolic equation is considered. Under the conditions $u(x, t)=X(x) T_{1}(t)+T_{2}(t), \frac{\partial u}{\partial x} \neq 0$, it is shown that the problem is equivalent to the system of two ordinary differential equations for which exact difference scheme (EDS) with special Steklov averaging and difference schemes with arbitrary order of accuracy (ADS) are constructed on the moving mesh. The special attention is paid to investigating approximation, stability and convergence of the ADS. The convergence of the iteration method is also considered. The presented numerical examples illustrate theoretical results investigated in the paper.
\end{abstract}

\section{Introduction}

In which cases an EDS or an ADS approximating nonlinear parabolic equation can be constructed? The paper deals with this question. The simple technique is presented and the main features of the constructed scheme are considered.

Definition 2. [3] A difference scheme is exact if the truncation error is equal to zero or the exact solution agrees with the numerical solution at the grid nodes.

The problem of constructing a difference scheme of high order of accuracy is topical. In papers $[1,6]$ the EDSs and truncated difference schemes of an arbitrary rank were constructed for the nonlinear second order differential equation and for the systems of first-order nonlinear

*lapkam@kul.pl 
ordinary differential equations, respectively. It is worth mentioning here the paper by Mickens [9] in which nonstandard finite difference schemes are introduced. In [10] the investigations of the order of approximation, stability, and convergence of the high accuracy difference schemes for the nonlinear transfer equation $\frac{\partial u}{\partial t}+u \frac{\partial u}{\partial x}=f(u)$ have been made. The EDS and the difference schemes of an arbitrary order of approximation for the parabolic equations with travelling wave solutions $u(x, t)=U(x-a t)$ were constructed in [7, 8].

It was earlier established that for the problems in the parabolic equations with separated variables solutions $u(x, t)=X(x) T_{1}(t)+T_{2}(t)$ the EDS and the ADSs may be constructed $[3,7]$. The main aim of this paper is to investigate approximation, stability and convergence of the nonlinear scheme of an arbitrary order of accuracy.

Let $C_{n}^{m}\left(\bar{Q}_{T}\right)$ be the class of functions with $m$ derivatives in $x$ and $n$ derivatives in $t$, all derivatives being continuous in the domain $\bar{Q}_{T}$, where $\bar{Q}_{T}=\mathbb{R} \times[0, T]$.

Let us consider the Cauchy problem for the semilinear parabolic equation

$$
\begin{gathered}
\frac{\partial u}{\partial t}=\frac{\partial}{\partial x}\left(k_{1}(x) k_{2}(t) \frac{\partial u}{\partial x}\right)+f(u), x \in \mathbb{R}, 0<t \leq T, \\
u(x, 0)=u_{0}(x), x \in \mathbb{R} .
\end{gathered}
$$

Under the conditions $u(x, t)=X(x) T_{1}(t)+T_{2}(t), \frac{\partial u}{\partial x} \neq 0$, we show that problem (1)-(2) is equivalent to the system of two ordinary differential equations [7]

$$
\begin{gathered}
\frac{d x}{d t}=c(x) k_{2}(t), x(0) \in \mathbb{R}, \quad c(x)=-\frac{\left(k_{1}(x) u_{0}^{\prime}(x)\right)^{\prime}}{u_{0}^{\prime}(x)}, \\
\left.\frac{d u}{d t}\right|_{\frac{d x}{d t}=c(x) k_{2}(t)}=f(u), u(x(0), 0)=u_{0}(x(0)) .
\end{gathered}
$$

From (3) we find the curve $x=x(t)$, along which we get from (4) the solution $u(x, t)=$ $u(x(t), t)$ of the problem (1)-(2). Here $x(0)=x^{0} \in \mathbb{R}$ is the initial state of the curve $x=x(t)$.

The ADS applying the trapezoidal rule is proposed in the case when the integrals in the EDS cannot be evaluated exactly. We prove that the orders of approximation and accuracy depend only on time step $\tau$ divided by the natural constant $m \geq 1$. We also consider the convergence of the iteration method which is used to find the solution of the nonlinear scheme. At the end we present the results of the numerical experiments which illustrate theoretical results stated in the paper. We also construct a difference scheme of an arbitrary order of accuracy applying the Euler-Maclaurin formula instead of the trapezoidal rule [2]. We investigate numerically that the error of this method equals $O\left(\left(\frac{\tau}{m}\right)^{2 M+2}\right)$, where $m, M$ are positive, natural constants.

\section{Statement of the problem}

Let us investigate in the domain $\bar{Q}_{T}$ the Cauchy problem for the semilinear parabolic equation 


$$
\begin{gathered}
\frac{\partial u}{\partial t}=\frac{\partial}{\partial x}\left(k_{1}(x) k_{2}(t) \frac{\partial u}{\partial x}\right)+f(u), x \in \mathbb{R}, 0<t \leq T, \\
u(x, 0)=u_{0}(x), x \in \mathbb{R}, \sup _{x \in \mathbb{R}}\left|u_{0}\right|<\infty, \\
u(x, t)=X(x) T_{1}(t)+T_{2}(t), \frac{\partial u}{\partial x} \neq 0,(x, t) \in \bar{Q}_{T}, \\
0<m_{1} \leq k_{1}(x) k_{2}(t) \leq m_{2},(x, t) \in \bar{Q}_{T}, m_{1}, m_{2}=\text { const, } f(u) \neq 0, u \in \mathbb{R} .
\end{gathered}
$$

Let us assume that problem (5) - (8) has a unique solution $u \in C_{1}^{4}\left(\bar{Q}_{T}\right)$ with all derivatives bounded and $k_{1}(x) \in C^{3}(\mathbb{R}), k_{2}(t) \in C^{2}([0, T]), f(u) \in C^{2}(\mathbb{R})$. Assumption (7) is the key relation. Taking into account these assumptions, we rewrite problem (5)-(6) in the following form

$$
\begin{gathered}
\frac{d x}{d t}=-\frac{\frac{\partial}{\partial x}\left(k_{1}(x) k_{2}(t) \frac{\partial u}{\partial x}\right)}{\frac{\partial u}{\partial x}}=-\frac{\left(k(x) u_{0}^{\prime}(x)\right)^{\prime}}{u_{0}^{\prime}(x)} k_{2}(t)=c(x) k_{2}(t), x(0) \in \mathbb{R}, \\
\left.\frac{d u}{d t}\right|_{\frac{d x}{d t}=c(x) k_{2}(t)}=\frac{\partial u}{\partial t}+\frac{d x}{d t} \frac{\partial u}{\partial t}=f(u), u(x(0), 0)=u_{0}(x(0)) .
\end{gathered}
$$

We assume that $c(x) \neq 0, x \in \mathbb{R}, c(x) \in C^{2}(\mathbb{R})$.

\section{The difference scheme of an arbitrary order of accuracy}

In this Section, the EDS approximating problem (5) - (8) is considered. The ADS is constructed in the case when the integrals in the EDS cannot be evaluated exactly. The trapezoidal rule is applied to approximate the integrals.

Let us introduce space and time grids

$$
\bar{\omega}_{h}^{0}=\left\{x_{i}^{0}=i h_{i}^{0}, i \in \mathbb{Z}\right\}, \bar{\omega}_{\tau}=\left\{t_{n}=n \tau, n=\overline{0, N_{0}}, \tau=\frac{T}{N_{0}}\right\},
$$

here $h_{i}^{n}=x_{i+1}^{n}-x_{i}^{n}$ is the space step at time $t=t_{n}$.

In $[3,7]$ the EDS approximating problem (5) - (8) was constructed

$$
\begin{gathered}
\frac{x_{i}^{n+1}-x_{i}^{n}}{\tau}=\left(\frac{1}{x_{i}^{n+1}-x_{i}^{n}} \int_{x_{i}^{n}}^{x_{i}^{n+1}} \frac{d x}{c(x)}\right)^{-1} \frac{1}{\tau} \int_{t_{n}}^{t_{n+1}} k_{2}(t) d t, \\
x_{i}^{0} \in \bar{\omega}_{h}^{0}, i \in \mathbb{Z}, n=\overline{0, N_{0}-1}, \\
\frac{y_{i}^{n+1}-y_{i}^{n}}{\tau}=\left(\frac{1}{y_{i}^{n+1}-y_{i}^{n}} \int_{y_{i}^{n}}^{y_{i}^{n+1}} \frac{d u}{f(u)}\right)^{-1}, y_{i}^{0}=u_{0}\left(x_{i}^{0}\right), i \in \mathbb{Z}, n=\overline{0, N_{0}-1} .
\end{gathered}
$$


Only in some cases the integrals in the EDS can be evaluated exactly. In other cases, applying the trapezoidal rule, we approximate problem (9) - (10) by the difference scheme

$$
\begin{gathered}
\frac{x_{h i}^{n+1}-x_{h i}^{n}}{\tau}=\varphi_{1}\left(x_{h i}^{n}, x_{h i}^{n+1}\right) \varphi_{2}\left(t_{n}, t_{n+1}\right), x_{h i}^{0}=x_{i}^{0} \in \bar{\omega}_{h}^{0}, i \in \mathbb{Z}, n=\overline{0, N_{0}-1}, \\
\frac{y_{i}^{n+1}-y_{i}^{n}}{\tau}=\varphi_{3}\left(y_{i}^{n}, y_{i}^{n+1}\right), y_{i}^{0}=u_{0}\left(x_{h i}^{0}\right), i \in \mathbb{Z}, n=\overline{0, N_{0}-1},
\end{gathered}
$$

where $m \geq 1$ is a natural number and $\varphi_{1}, \varphi_{2}, \varphi_{3}: \mathbb{R}^{2} \rightarrow \mathbb{R}$ are introduced as follows

$$
\begin{aligned}
& \varphi_{1}\left(s_{1}, s_{2}\right)=\left[\frac{1}{2 m}\left(\frac{1}{c\left(s_{1}\right)}+\frac{1}{c\left(s_{2}\right)}\right)+\frac{1}{m} \sum_{j=1}^{m-1} \frac{1}{c\left(s_{1}+j \frac{s_{2}-s_{1}}{m}\right)}\right]^{-1}, \\
& \varphi_{2}\left(s_{1}, s_{2}\right)=\left(k_{2}\left(s_{1}\right)+k_{2}\left(s_{2}\right)\right) /(2 m)+\sum_{j=1}^{m-1} k_{2}\left(s_{1}+j \frac{s_{2}-s_{1}}{m}\right) / m, \\
& \varphi_{3}\left(s_{1}, s_{2}\right)=\left[\frac{1}{2 m}\left(\frac{1}{f\left(s_{1}\right)}+\frac{1}{f\left(s_{2}\right)}\right)+\frac{1}{m} \sum_{j=1}^{m-1} \frac{1}{f\left(s_{1}+j \frac{s_{2}-s_{1}}{m}\right)}\right]^{-1} .
\end{aligned}
$$

Let us denote the approximate value of the curve $x_{i}(t)$ on $n-t h$ level by $x_{h i}^{n}$, and the exact value of this curve on $n-t h$ level by $x_{i}^{n}$. The curve $x_{i}(t)$ is the solution of problem (9) with the initial value $x_{i}^{0}$. Here $y_{i}^{n}$ is the approximate solution of problem (10) in the node $\left(x_{h i}^{n}, t_{n}\right)$ and $u_{i}^{n}, u_{h i}^{n}$ are the values of exact solution of this problem in the nodes $\left(x_{i}^{n}, t_{n}\right)$ and $\left(x_{h i}^{n}, t_{n}\right)$, respectively.

Equation (13) introduces the moving grid in the domain $\bar{Q}_{T}$

$$
\bar{\omega}=\left\{\left(x_{h i}^{n}, t_{n}\right) \in \bar{Q}_{T}: x_{h i}^{0}=i h_{i}^{0}, i \in \mathbb{Z}, t_{n}=n \tau, n=\overline{0, N_{0}}\right\} .
$$

Let us denote the error of the method by

$$
\delta x_{h i}^{n}=x_{h i}^{n}-x_{i}^{n}, \delta u_{h i}^{n}=y_{i}^{n}-u_{h i}^{n} .
$$

Then the difference problem for the error of the method is written in the following form 


$$
\begin{gathered}
\frac{\delta x_{h i}^{n+1}-\delta x_{h i}^{n}}{\tau}-\delta x_{h i}^{n} \varphi_{2}\left(t_{n}, t_{n+1}\right) \frac{\partial \varphi_{1}}{\partial s_{1}}\left(x_{\theta 1 i}^{n}, x_{\theta 2 i}^{n+1}\right) \\
-\delta x_{h i}^{n+1} \varphi_{2}\left(t_{n}, t_{n+1}\right) \frac{\partial \varphi_{1}}{\partial s_{2}}\left(x_{\theta 1 i}^{n}, x_{\theta 2 i}^{n+1}\right)=\psi_{1 i}^{n}, \\
\delta x_{h i}^{0}=0, i \in \mathbb{Z}, n=\overline{0, N_{0}-1}, \\
\frac{\delta u_{h i}^{n+1}-\delta u_{h i}^{n}}{\tau}-\delta u_{h i}^{n} \frac{\partial \varphi_{3}}{\partial s_{1}}\left(u_{\theta 3 i}^{n}, u_{\theta 4 i}^{n+1}\right)-\delta u_{h i}^{n+1} \frac{\partial \varphi_{3}}{\partial s_{2}}\left(u_{\theta 3 i}^{n}, u_{\theta 4 i}^{n+1}\right) \\
+\delta x_{h i}^{n+1} \frac{\partial u}{\partial x}\left(x_{\theta 6 i}^{n+1}, t_{n+1}\right)\left(\frac{1}{\tau}-\frac{\partial \varphi_{3}}{\partial s_{2}}\left(u_{\theta 3 i}^{n}, u_{\theta 4 i}^{n+1}\right)\right) \\
-\delta x_{h i}^{n} \frac{\partial u}{\partial x}\left(x_{\theta 5 i}^{n}, t_{n}\right)\left(\frac{1}{\tau}+\frac{\partial \varphi_{3}}{\partial s_{1}}\left(u_{\theta 3 i}^{n}, u_{\theta 4 i}^{n+1}\right)\right)=\psi_{2 i}^{n}, \\
\delta u_{h i}^{0}=0, i \in \mathbb{Z}, n=\overline{0, N_{0}-1},
\end{gathered}
$$

where $x_{\theta p i}^{n}=x_{i}^{n}+\theta_{p i}^{n} \delta x_{h i}^{n}, u_{\theta p i}^{n}=u_{i}^{n}+\theta_{p i}^{n}\left(\delta u_{h i}^{n}+u_{h i}^{n}-u_{i}^{n}\right)$. Here and after $\theta_{p i}^{n}=$ const, $0<\theta_{p i}^{n}<1$. The approximation error of the difference scheme (13) - (14) equals

$$
\psi_{i}^{n}=\psi_{1 i}^{n}+\psi_{2 i}^{n},
$$

where $\psi_{1 i}^{n}=-\frac{x_{h i}^{n+1}-x_{h i}^{n}}{\tau}+\varphi_{1}\left(x_{i}^{n}, x_{i}^{n+1}\right) \varphi_{2}\left(t_{n}, t_{n+1}\right), \quad \psi_{2 i}^{n}=-\frac{u_{i}^{n+1}-u_{i}^{n}}{\tau}+\varphi_{3}\left(u_{i}^{n}, u_{i}^{n+1}\right)$.

\section{Approximation}

In this Section, we prove the theorem on an order of approximation of difference scheme (13) - (14).

Theorem 2. Suppose that

$$
\begin{gathered}
c(x) \in C^{2}(\mathbb{R}), 0<c_{1} \leq\left|\frac{1}{c(x)}\right| \leq c_{2},\left|\left(\frac{1}{c(x)}\right)^{(2)}\right| \leq c_{3}, x \in \mathbb{R}, \\
k_{2}(t) \in C^{2}([0, T]),\left|k_{2}(t)\right| \leq c_{4},\left|k_{2}^{\prime \prime}(t)\right| \leq c_{5}, t \in[0, T], \\
f(u) \in C^{2}(\mathbb{R}), 0<c_{6} \leq\left|\frac{1}{f(u)}\right| \leq c_{7},\left|\left(\frac{1}{f(u)}\right)^{(2)}\right| \leq c_{8}, u \in \mathbb{R}, \\
\left\|\frac{\partial u}{\partial t}\right\|_{C\left(\bar{Q}_{T}\right)} \leq c_{9},
\end{gathered}
$$

where constants $c_{p}>0$. Then difference scheme (13)-(14) approximates problem (9)-(10) with the truncation error equal to $O\left(\left(\frac{\tau}{m}\right)^{2}\right)$. 
PROOF. Our first goal is to show that $\psi_{1 i}^{n}=R_{i}^{n}\left(\frac{\tau}{m}\right)^{2}$, where the expression $\left|R_{i}^{n}\right|$ is bounded. It is straightforward to show that the approximation error $\psi_{1 i}^{n}$ satisfies the chain of relations

$$
\begin{gathered}
\psi_{1 i}^{n}=\left[\frac{-1}{x_{i}^{n+1}-x_{i}^{n}} \int_{x_{i}^{n}}^{x_{i}^{n+1}} \frac{d x}{c(x)}\right]^{-1} \frac{1}{\tau} \int_{t_{n}}^{t_{n+1}} k_{2}(t) d t+\varphi_{1}\left(x_{i}^{n}, x_{i}^{n+1}\right) \varphi_{2}\left(t_{n}, t_{n+1}\right) \\
=-\frac{x_{i}^{n+1}-x_{i}^{n}}{m}\left[\frac{1}{2 c\left(x_{i}^{n}\right)}+\frac{1}{2 c\left(x_{i}^{n+1}\right)}+\sum_{j=1}^{m-1} \frac{1}{c\left(x_{i}^{n+j} \frac{x_{i}^{n+1}-x_{i}^{n}}{m}\right)}\right]-\int_{x_{i}^{n}}^{x_{i}^{n+1}} \frac{d x}{c(x)}{ }^{t_{n+1}} \int_{t_{n}}^{t_{n+1}} k_{2}(t) d t \\
{\left[\int_{x_{i}^{n}}^{x_{i}^{n+1}} \frac{d x}{c(x)}\right]\left[\varphi_{1}\left(x_{i}^{n}, x_{i}^{n+1}\right)\right]^{-1}} \\
+\frac{1}{12}\left(\frac{\tau}{m}\right)^{2} k_{2}^{\prime \prime}\left(t_{\theta 7 n}\right) \varphi_{1}\left(x_{i}^{n}, x_{i}^{n+1}\right)=\left(\frac{\tau}{m}\right)^{2} R_{i}^{n},
\end{gathered}
$$

where $i \in \mathbb{Z}, n=\overline{0, N_{0}-1}$ and

$$
\begin{gathered}
R_{i}^{n}=-\frac{\frac{x_{i}^{n+1}-x_{i}^{n}}{12}\left(\frac{d x\left(t_{\theta 8 n}\right)}{d t}\right)^{2}\left(\frac{1}{c\left(x_{\theta 9 i}\right)}\right)^{(2)}}{\left[\int_{x_{i}^{n}}^{x_{i}^{n+1}} \frac{d x}{c(x)}\right]\left[\varphi_{1}\left(x_{i}^{n}, x_{i}^{n+1}\right)\right]^{-1}} \int_{t_{n}}^{t_{n+1}} k_{2}(t) d t+\frac{1}{12} k_{2}^{\prime \prime}\left(t_{\theta 7 n}\right) \varphi_{1}\left(x_{i}^{n}, x_{i}^{n+1}\right), \\
x_{\theta 9 i}=x_{i}^{n}+\theta_{9 i}^{n}\left(x_{i}^{n+1}-x_{i}^{n}\right), t_{\theta 7 n}, t_{\theta 8 n} \in\left(t_{n}, t_{n+1}\right) .
\end{gathered}
$$

Since (16), the function $\frac{1}{c(x)}$ has a constant sign for all $x \in \mathbb{R}$. Thus, the following estimate is valid

$$
\left|\left[\varphi_{1}\left(x_{i}^{n}, x_{i}^{n+1}\right)\right]^{-1}\right|=\frac{1}{m}\left\{\left|\frac{1}{2 c\left(x_{i}^{n}\right)}\right|+\left|\frac{1}{2 c\left(x_{i}^{n+1}\right)}\right|+\sum_{j=1}^{m-1}\left|\frac{1}{c\left(x_{i}^{n}+j \frac{x_{i}^{n+1}-x_{i}^{n}}{m}\right)}\right|\right\} \geq c_{1} .
$$

Some tedious manipulations yields

$$
\left|R_{i}^{n}\right| \leq M_{A}, M_{A}=\left(c_{1}^{-4} c_{3} c_{4}^{3}+c_{1}^{-1} c_{5}\right) / 12 .
$$

Thus, difference equation (13) approximates differential problem (9) with the second order with respect to $\frac{\tau}{m}$

$$
\max _{0 \leq n<N_{0}}\left\|\psi_{1}^{n}\right\|_{C} \leq M_{A}\left(\frac{\tau}{m}\right)^{2},\left\|\psi_{1}^{n}\right\|_{C}=\sup _{i \in \mathbb{Z}}\left|\psi_{1 i}^{n}\right|
$$

Analogously, we can show that difference equation (14) approximates differential problem (10) with the second order with respect to $\frac{\tau}{m}$

$$
\max _{0 \leq n<N_{0}}\left\|\psi_{2}^{n}\right\|_{C} \leq M_{A}^{\prime}\left(\frac{\tau}{m}\right)^{2}, M_{A}^{\prime}=c_{6}^{-2} c_{8} c_{9}^{2} / 12 .
$$


Finally, we find that difference scheme (13) - (14) approximates differential problem (9) (10) with the second order with respect to $\frac{\tau}{m}$

$$
\max _{0 \leq n<N_{0}}\left\|\psi^{n}\right\|_{C} \leq\left(M_{A}+M_{A}^{\prime}\right)\left(\frac{\tau}{m}\right)^{2} .
$$

\section{Stability}

In this Section, stability of the ADS is investigated. Let us perturb the initial data of problem (13) - (14)

$$
\begin{gathered}
\frac{\tilde{x}_{h i}^{n+1}-\tilde{x}_{h i}^{n}}{\tau}=\varphi_{1}\left(\tilde{x}_{h i}^{n}, \tilde{x}_{h i}^{n+1}\right) \varphi_{2}\left(t_{n}, t_{n+1}\right), \tilde{x}_{h i}^{0}=\tilde{x}_{i}^{0}, i \in \mathbb{Z}, n=\overline{0, N_{0}-1} \\
\frac{\tilde{y}_{i}^{n+1}-\tilde{y}_{i}^{n}}{\tau}=\varphi_{3}\left(\tilde{y}_{i}^{n}, \tilde{y}_{i}^{n+1}\right), \tilde{y}_{i}^{0}=\tilde{u}_{0}\left(\tilde{x}_{h i}^{0}\right), i \in \mathbb{Z}, n=\overline{0, N_{0}-1}
\end{gathered}
$$

We give a theorem on stability of difference scheme (13)-(14).

Theorem 3. Suppose that

$$
\begin{gathered}
c(x) \in C^{1}(\mathbb{R}), 0<c_{1} \leq\left|\frac{1}{c(x)}\right| \leq c_{2},\left|\left(\frac{1}{c(x)}\right)^{(1)}\right| \leq c_{10}, x \in \mathbb{R}, \\
k_{2}(t) \in C^{1}([0, T]),\left|k_{2}(t)\right| \leq c_{4}, t \in[0, T] \\
f(u) \in C^{1}(\mathbb{R}), 0<c_{6} \leq\left|\frac{1}{f(u)}\right| \leq c_{7},\left|\left(\frac{1}{f(u)}\right)^{(1)}\right| \leq c_{11}, u \in \mathbb{R},
\end{gathered}
$$

where the constants $c_{p}>0$. Then for sufficiently small $\tau \leq \min \left\{\tau_{0}^{*}, \tau_{0}^{* *}\right\}$ difference scheme (13)-(14) is stable with respect to small perturbation of the initial values and the following estimates hold

$$
\begin{gathered}
\max _{0 \leq n \leq N_{0}}\left\|\tilde{x}_{h}^{n}-x_{h}^{n}\right\|_{C} \leq e^{T\left(M_{2}+2 M_{1}\right)}\left\|\tilde{x}_{h}^{0}-x_{h}^{0}\right\|_{C}, M_{1}, M_{2}=\text { const }>0 \\
\max _{0 \leq n \leq N_{0}}\left\|\tilde{y}^{n}-y^{n}\right\|_{C} \leq e^{T\left(M_{4}+2 M_{3}\right)}\left\|\tilde{u}_{0}-u_{0}\right\|_{C}, M_{3}, M_{4}=\text { const }>0 .
\end{gathered}
$$

Proof. Under assumptions (22), (24), the functions $\varphi_{1}, \varphi_{3} \in C^{1}\left(\mathbb{R}^{2}\right)$ have bounded derivatives

$$
\begin{aligned}
& \left|\frac{\partial \varphi_{1}}{\partial s_{2}}\left(s, s_{2}\right)\right| \leq M_{1},\left|\frac{\partial \varphi_{1}}{\partial s_{1}}\left(s_{1}, s_{2}\right)\right| \leq M_{2},\left(s_{1}, s_{2}\right) \in \mathbb{R}^{2}, \\
& \left|\frac{\partial \varphi_{3}}{\partial s_{2}}\left(s, s_{2}\right)\right| \leq M_{3},\left|\frac{\partial \varphi_{3}}{\partial s_{1}}\left(s_{1}, s_{2}\right)\right| \leq M_{4},\left(s_{1}, s_{2}\right) \in \mathbb{R}^{2}
\end{aligned}
$$

where $M_{p}$ are positive constants. 
Subtracting equations (13) - (14) from (20) - (21) and introducing the notation $\Delta x_{h i}^{n}=$ $\tilde{x}_{h i}^{n}-x_{h i}^{n}, \Delta y_{i}^{n}=\tilde{y}_{i}^{n}-y_{i}^{n}$, we get

$$
\begin{gathered}
\frac{\Delta x_{h i}^{n+1}-\Delta x_{h i}^{n}}{\tau}=\left(\Delta x_{h i}^{n} \frac{\partial \varphi_{1}}{\partial s_{1}}\left(x_{\theta 10 i}^{n}, x_{\theta 11 i}^{n+1}\right)+\Delta x_{h i}^{n+1} \frac{\partial \varphi_{1}}{\partial s_{2}}\left(x_{\theta 10 i}^{n}, x_{\theta 11 i}^{n+1}\right)\right) \varphi_{2}\left(t_{n}, t_{n+1}\right), \\
\Delta x_{h i}^{0}=\tilde{x}_{i}^{0}-x_{i}^{0}, i \in \mathbb{Z}, n=\overline{0, N_{0}-1}, \\
\frac{\Delta y_{i}^{n+1}-\Delta y_{i}^{n}}{\tau}=\Delta y_{i}^{n} \frac{\partial \varphi_{3}}{\partial s_{1}}\left(y_{\theta 12 i}^{n}, y_{\theta 13 i}^{n+1}\right)+\Delta y_{i}^{n+1} \frac{\partial \varphi_{3}}{\partial s_{2}}\left(y_{\theta 12 i}^{n}, y_{\theta 13 i}^{n+1}\right), \\
\Delta y_{i}^{0}=\tilde{u}_{0}\left(\tilde{x}_{h i}^{0}\right)-u_{0}\left(x_{h i}^{0}\right), i \in \mathbb{Z}, n=\overline{0, N_{0}-1},
\end{gathered}
$$

where $x_{\theta p i}^{n}=x_{h i}^{n}+\theta_{p i}^{n}\left(\tilde{x}_{h i}^{n}-x_{h i}^{n}\right), y_{\theta p i}^{n}=y_{i}^{n}+\theta_{p i}^{n}\left(\tilde{y}_{i}^{n}-y_{i}^{n}\right)$. The following grid node's estimate is valid

$$
\begin{gathered}
\left|\Delta x_{h i}^{n+1}\right| \leq \tau\left|\frac{\partial \varphi_{1}}{\partial s_{2}}\left(x_{\theta 10 i}^{n}, x_{\theta 11 i}^{n+1}\right)\right|\left|\varphi_{2}\left(t_{n}, t_{n+1}\right)\right|\left|\Delta x_{h i}^{n+1}\right|+ \\
+\left(1+\tau\left|\frac{\partial \varphi_{1}}{\partial s_{1}}\left(x_{\theta 10 i}^{n}, x_{\theta 11 i}^{n+1}\right)\right|\left|\varphi_{2}\left(t_{n}, t_{n+1}\right)\right|\right)\left|\Delta x_{h i}^{n}\right| \\
\left(1-\tau M_{1} c_{4}\right)\left|\Delta x_{h i}^{n+1}\right| \leq\left(1+\tau M_{2} c_{4}\right)\left|\Delta x_{h i}^{n}\right| \leq\left(1+\tau M_{2} c_{4}\right)\left\|\Delta x_{h}^{n}\right\|_{C} \leq e^{\tau M_{2} c_{4}}\left\|\Delta x_{h}^{n}\right\|_{C} .
\end{gathered}
$$

For sufficiently small $\tau \leq \tau_{0}^{*}$, where $\tau_{0}^{*}=\frac{1}{2 M_{1} c_{4}}$, the following inequalities hold

$$
\left|\Delta x_{h i}^{n+1}\right| \leq \frac{e^{\tau M_{2} c_{4}}}{1-\tau M_{1} c_{4}}\left\|\Delta x_{h}^{n}\right\|_{C} \leq e^{\tau\left(M_{2}+2 M_{1}\right) c_{4}}\left\|\Delta x_{h}^{n}\right\|_{C} .
$$

Thus

$$
\left\|\Delta x_{h}^{n+1}\right\|_{C} \leq e^{\tau\left(M_{2}+2 M_{1}\right) c_{4}}\left\|\Delta x_{h}^{n}\right\|_{C} \leq \cdots \leq e^{(n+1) \tau\left(M_{2}+2 M_{1}\right) c_{4}}\left\|\Delta x_{h}^{0}\right\|_{C} .
$$

Because it is satisfied for any $n=\overline{0, N_{0}-1}$, then

$$
\max _{0 \leq n \leq N_{0}}\left\|\Delta x_{h}^{n}\right\|_{C} \leq e^{T\left(M_{2}+2 M_{1}\right) c_{4}}\left\|\Delta x_{h}^{0}\right\|_{C}
$$

Analogously, we can prove that for sufficiently small $\tau \leq \tau_{0}^{* *}$, where $\tau_{0}^{* *}=\frac{1}{2 M_{3}}$

$$
\max _{0 \leq n \leq N_{0}}\left\|\Delta y^{n}\right\|_{C} \leq e^{T\left(M_{4}+2 M_{3}\right)}\left\|\Delta u_{0}\right\|_{C}
$$

Estimates (25), (26) express the stability of difference scheme (13) - (14) with respect to the small perturbation of the initial values.

\section{Convergence}

In this Section, we prove the theorem on an order of accuracy of difference scheme (13) (14). 
Theorem 4. Let the assumptions of Theorems 2 and 3 holds. Then for sufficiently small $\tau \leq \min \left\{\tau_{0}^{*}, \tau_{0}^{* *}\right\}$ the solution of the difference scheme (13) - (14) converges to the solution of the differential problem (9) - (10) and the estimates hold

$$
\begin{aligned}
& \max _{0 \leq n \leq N_{0}}\left\|x_{h}^{n}-x^{n}\right\|_{C} \leq M_{Z}\left(\frac{\tau}{m}\right)^{2}, M_{Z}=\text { const }>0, \\
& \max _{0 \leq n \leq N_{0}}\left\|y^{n}-u_{h}^{n}\right\|_{C} \leq M_{Z}^{\prime}\left(\frac{\tau}{m}\right)^{2}, M_{Z}^{\prime}=\text { const }>0 .
\end{aligned}
$$

PROOF. Similarly to that before, the functions $\varphi_{1}, \varphi_{3} \in C^{1}\left(\mathbb{R}^{2}\right)$ have bounded derivatives

$$
\begin{aligned}
& \left|\frac{\partial \varphi_{1}}{\partial s_{2}}\left(s, s_{2}\right)\right| \leq M_{1},\left|\frac{\partial \varphi_{1}}{\partial s_{1}}\left(s_{1}, s_{2}\right)\right| \leq M_{2},\left(s_{1}, s_{2}\right) \in \mathbb{R}^{2}, \\
& \left|\frac{\partial \varphi_{3}}{\partial s_{2}}\left(s, s_{2}\right)\right| \leq M_{3},\left|\frac{\partial \varphi_{3}}{\partial s_{1}}\left(s_{1}, s_{2}\right)\right| \leq M_{4},\left(s_{1}, s_{2}\right) \in \mathbb{R}^{2},
\end{aligned}
$$

where $M_{p}$ are positive constants. From the equation for the error of the method the following estimates hold

$$
\begin{aligned}
& \left|\delta x_{h i}^{n+1}\right| \leq \tau\left|\frac{\partial \varphi_{1}}{\partial s_{2}}\left(x_{\theta 1 i}^{n}, x_{\theta 2 i}^{n+1}\right)\right|\left|\varphi_{2}\left(t_{n}, t_{n+1}\right)\right|\left|\delta x_{h i}^{n+1}\right|+ \\
& +\left|1+\tau \frac{\partial \varphi_{1}}{\partial s_{1}}\left(x_{\theta 1 i}^{n}, x_{\theta_{2} i}^{n+1}\right)\right|\left|\varphi_{3}\left(t_{n}, t_{n+1}\right)\right|\left|\delta x_{h i}^{n}\right|+\tau\left|\psi_{1 i}^{n}\right| \\
& \left(1-\tau M_{1} c_{4}\right)\left|\delta x_{h i}^{n+1}\right| \leq\left(1+\tau M_{2} c_{4}\right)\left\|\delta x_{h}^{n}\right\|_{C}+\tau\left\|\psi_{1}^{n}\right\|_{C} .
\end{aligned}
$$

For sufficiently small $\tau \leq \tau_{0}^{*}$, where $\tau_{0}^{*}=\frac{1}{2 M_{1} c_{4}}$, we have

$$
\begin{gathered}
\left|\delta x_{h i}^{n+1}\right| \leq \frac{e^{\tau M_{2} c_{4}}}{1-\tau M_{1} c_{4}}\left\|\delta x_{h}^{n}\right\|_{C}+\frac{\tau}{1-\tau M_{1} c_{4}}\left\|\psi_{1}^{n}\right\|_{C} \leq \\
\leq e^{\tau\left(M_{2}+2 M_{1}\right) c_{4}}\left\|\delta x_{h}^{n}\right\|_{C}+\tau e^{2 \tau M_{1} c_{4}}\left\|\psi_{1}^{n}\right\|_{C} .
\end{gathered}
$$

The above inequalities are valid for any $i \in \mathbb{Z}$, thus

$$
\begin{gathered}
\left\|\delta x_{h}^{n+1}\right\|_{C} \leq e^{\tau\left(M_{2}+2 M_{1}\right) c_{4}}\left\|\delta x_{h}^{n}\right\|_{C}+\tau e^{2 \tau M_{1} c_{4}}\left\|\psi_{1}^{n}\right\|_{C} \leq \\
\leq \cdots \leq e^{(n+1) \tau\left(M_{2}+2 M_{1}\right) c_{4}}\left\|\delta x_{h}^{0}\right\|_{C}+\tau \sum_{k=0}^{n} e^{(n-k) \tau\left(M_{2}+2 M_{1}\right) c_{4}+2 \tau M_{1} c_{4}}\left\|\psi_{1}^{k}\right\|_{C} \leq \\
\leq \max _{0 \leq k \leq n}\left\|\psi_{1}^{k}\right\|_{C} t_{n+1} e^{T\left(M_{2}+4 M_{1}\right) c_{4}} .
\end{gathered}
$$

Since $\psi_{1 i}^{n}=O\left(\left(\frac{\tau}{m}\right)^{2}\right)$, then

$$
\max _{0 \leq n \leq N_{0}}\left\|\delta x_{h}^{n}\right\|_{C} \leq M_{Z}\left(\frac{\tau}{m}\right)^{2}, M_{Z}=e^{T\left(M_{2}+4 M_{1}\right) c_{4}} T M_{A} .
$$

To obtain the estimation of the error $\delta u_{h i}^{n}$ a little manipulation is needed 


$$
\left\|\delta u_{h}^{n+1}\right\|_{C} \leq\left\|\delta y^{n+1}\right\|_{C}+\left\|u^{n+1}-u_{h}^{n+1}\right\|_{C} \leq\left\|\delta y^{n+1}\right\|_{C}+\left\|\delta x_{h}^{n+1}\right\|_{C}\left\|\frac{\partial u}{\partial x}\right\|_{C\left(\bar{Q}_{T}\right)},
$$

where $\delta y_{i}^{n}=y_{i}^{n}-u_{i}^{n}$ satisfies the equation

$$
\begin{gathered}
\frac{\delta y_{i}^{n+1}-\delta y_{i}^{n}}{\tau}-\delta y_{i}^{n} \frac{\partial \varphi_{3}}{\partial s_{1}}\left(y_{\theta 14 i}^{n}, y_{\theta 15 i}^{n+1}\right)-\delta y_{i}^{n+1} \frac{\partial \varphi_{3}}{\partial s_{2}}\left(y_{\theta 14 i}^{n}, y_{\theta 15 i}^{n+1}\right)=\psi_{2 i}^{n}, \\
y_{\theta 14 i}^{n}=u_{i}^{n}+\theta_{14 i}^{n} \delta y_{i}^{n}, y_{\theta 15 i}^{n+1}=u_{i}^{n+1}+\theta_{14 i}^{n+1} \delta y_{i}^{n+1} .
\end{gathered}
$$

Our problem reduces to estimating the error $\delta y_{i}^{n}$. A reasoning similar to that used in the first part of the proof, shows that

$$
\left\|\delta y^{n+1}\right\|_{C} \leq \max _{0 \leq k \leq n}\left\|\psi_{2}^{k}\right\|_{C} t_{n+1} e^{T\left(M_{4}+4 M_{3}\right)}
$$

We get

$$
\begin{gathered}
\max _{0 \leq n \leq N_{0}}\left\|\delta u_{h}^{n}\right\|_{C} \leq T e^{T\left(M_{4}+4 M_{3}\right)} \max _{0 \leq k \leq n}\left\|\psi_{2}^{k}\right\|_{C} \\
+T e^{T\left(M_{2}+4 M_{1}\right) c_{4}}\left\|\frac{\partial u}{\partial x}\right\|_{C\left(\bar{Q}_{T}\right)} \max _{0 \leq k \leq n}\left\|\psi_{1}^{k}\right\|_{C} \leq M_{Z}^{\prime}\left(\frac{\tau}{m}\right)^{2}, M_{Z}^{\prime} \\
=T e^{T\left(M_{4}+4 M_{3}\right)} M_{A}^{\prime}+T e^{T\left(M_{2}+4 M_{1}\right) c_{4}} M_{A}\left\|\frac{\partial u}{\partial x}\right\|_{C\left(\bar{Q}_{T}\right)} .
\end{gathered}
$$

Finally, estimates (27) and (28) express the convergence of the solution of difference scheme (13) - (14) to the solution of differential problem (9) - (10) with the second order with respect to $\frac{\tau}{m}$.

\section{Iteration method}

In this Section, convergence of the iteration method for difference scheme (13) - (14) is discussed.

The following iteration method is used in connection with solving nonlinear difference scheme (13) - (14)

$$
\begin{aligned}
& \frac{\stackrel{s+1}{x}_{h i}^{n+1}-x_{h i}^{n}}{\tau}=\varphi_{1}\left(x_{h i}^{n}, \stackrel{s}{x}_{h i}^{n+1}\right) \varphi_{2}\left(t_{n}, t_{n+1}\right),
\end{aligned}
$$

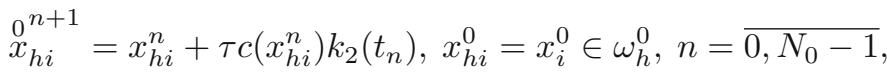

$$
\begin{aligned}
& \frac{{\stackrel{s+1}{y_{i}}}_{i}^{n+1}-y_{i}^{n}}{\tau}=\varphi_{3}\left(y_{i}^{n}, \stackrel{s}{y}_{i}^{n+1}\right), \\
& {y_{i}^{n+1}}^{n}=y_{i}^{n}+\tau f\left(y_{i}^{n}\right), y_{i}^{0}=u_{0}\left(x_{h i}^{0}\right), i \in \mathbb{Z}, n=\overline{0, N_{0}-1} \text {. }
\end{aligned}
$$


We give a theorem on convergence of iteration method (29) - (30).

Theorem 5. Let the assumptions of Theorem 4 holds. Then for sufficiently small $\tau \leq$ $\tau_{0}, \tau_{0} \leq \min \left\{\frac{q}{M_{1} c_{4}}, \frac{q}{M_{3}}\right\}, 0<q<1$ the solution of iteration method (29) - (30) converges to the solution of difference equation (13) - (14).

PROOF. Let $\tau$ be chosen sufficiently small

$$
\tau \leq \tau_{0}, \tau_{0} \leq \min \left\{\frac{q}{M_{1} c_{4}}, \frac{q}{M_{3}}\right\}, 0<q<1
$$

First, we estimate the difference between $\stackrel{1}{x}_{h i}^{n+1}$ and the initial approximation

$$
\begin{aligned}
& \left|\begin{array}{ll}
1^{n+1} & -0_{h i}^{n+1} \\
x_{h i}
\end{array}\right|=\left|x_{h i}^{n}+\tau \varphi_{1}\left(x_{h i}^{n}, \stackrel{0}{x}_{h i}^{n+1}\right) \varphi_{2}\left(t_{n}, t_{n+1}\right)-x_{h i}^{n}-\tau c\left(x_{h i}^{n}\right) k_{2}\left(t_{n}\right)\right| \\
& =\tau\left|\varphi_{1}\left(x_{h i}^{n}, \stackrel{0}{x}_{h i}^{n+1}\right) \varphi_{2}\left(t_{n}, t_{n+1}\right)-c\left(x_{h i}^{n}\right) k_{2}\left(t_{n}\right)\right| \leq 2 \tau_{0} c_{1}^{-1} c_{4} .
\end{aligned}
$$

Now, let us estimate the difference between two successive iterations

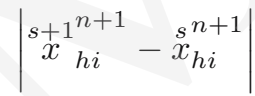

$$
\begin{aligned}
& =\left|x_{h i}^{n}+\tau \varphi_{1}\left(x_{h i}^{n}, \stackrel{s^{n+1}}{x_{h i}}\right) \varphi_{2}\left(t_{n}, t_{n+1}\right)-x_{h i}^{n}-\tau \varphi_{1}\left(x_{h i}^{n},{ }^{s-1} x_{h i}^{n+1}\right) \varphi_{2}\left(t_{n}, t_{n+1}\right)\right|
\end{aligned}
$$

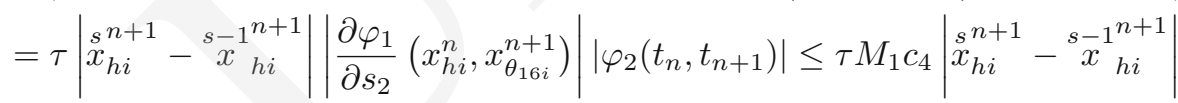

$$
\begin{aligned}
& \leq q\left|\begin{array}{l}
s^{n+1} \\
x_{h i}-{ }^{s} x_{h i}^{n+1}
\end{array}\right| \leq \cdots \leq q^{s}\left|\begin{array}{ll}
1^{n+1} & -0_{h i}^{n+1} \\
x_{h i}
\end{array}\right| \leq 2 \tau_{0} c_{1}^{-1} c_{4} q^{s} .
\end{aligned}
$$

where $x_{\theta_{16 i}}^{n+1}=\stackrel{s}{x}_{h i}^{n+1}+\theta_{16 i}^{n+1}\left(\stackrel{s}{x}_{h i}^{n+1}-\stackrel{s}{x}_{h i}^{n+1}\right)$. We are now in a position to estimate the error $x_{h i}^{n+1}-\stackrel{s}{x}_{h i}^{n+1}$

$$
\begin{aligned}
& \left|x_{h i}^{n+1}-\stackrel{s}{x}_{h i}^{n+1}\right| \leq\left|x_{h i}^{n+1}-x_{h i}^{n}-\tau \varphi_{1}\left(x_{h i}^{n}, x_{h i}^{n+1}\right) \varphi_{2}\left(t_{n}, t_{n+1}\right)\right| \\
& +\left|x_{h i}^{n}+\tau \varphi_{1}\left(x_{h i}^{n}, x_{h i}^{n+1}\right) \varphi_{2}\left(t_{n}, t_{n+1}\right)-x_{h i}^{n}-\tau \varphi_{1}\left(x_{h i}^{n}, x_{h i}^{n+1}\right) \varphi_{2}\left(t_{n}, t_{n+1}\right)\right| \\
& +\left|x_{h i}^{n}+\tau \varphi_{1}\left(x_{h i}^{n}, \stackrel{s^{n+1}}{h i}\right) \varphi_{2}\left(t_{n}, t_{n+1}\right)-\stackrel{s}{x}_{h i}^{n+1}\right|
\end{aligned}
$$

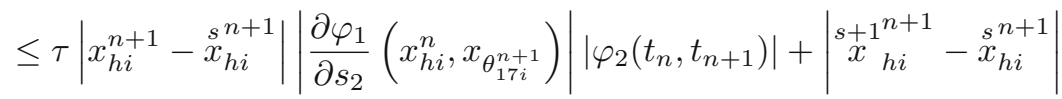

$$
\begin{aligned}
& \leq q\left|x_{h i}^{n+1}-\stackrel{s}{x}_{h i}^{n+1}\right|+\left|\begin{array}{ll}
s_{x}^{+1} & -\stackrel{s}{x+1}_{h i}^{n+1}
\end{array}\right| \text {. }
\end{aligned}
$$


where $x_{\theta_{17 i}}^{n+1}=x_{h i}^{n+1}+\theta_{17 i}^{n+1}\left(\stackrel{s}{x}_{h i}^{n+1}-x_{h i}^{n+1}\right)$. Thus we have

$$
\left|x_{h i}^{n+1}-\stackrel{s}{x}_{h i}^{n+1}\right| \leq \frac{1}{1-q}\left|\stackrel{s}{x}_{h i}^{n+1}-\stackrel{s}{x}_{h i}^{n+1}\right| \leq M_{I} q^{s}, M_{I}=2 \tau_{0} c_{1}^{-1} c_{4} /(1-q) .
$$

The above inequality is valid for any $i \in \mathbb{Z}$, so we get

$$
\left\|x_{h}^{n+1}-\stackrel{s}{x}_{h}^{n+1}\right\|_{C} \leq M_{I} q^{s} .
$$

It remains to show that

$$
\left\|y^{n+1}-\stackrel{s}{n+1}^{n}\right\|_{C} \leq M_{I}^{\prime} q^{s}, M_{I}^{\prime}=2 \tau_{0} c_{6}^{-1} /(1-q) .
$$

The proof is similar to that used above.

Thereby the iterations converge with the rate of geometric progression and the limits exist $\lim _{s \rightarrow \infty}\left\|x_{h}^{n+1}-x_{h}^{n+1}\right\|_{C}=0, \lim _{s \rightarrow \infty}\left\|y^{n+1}-y^{n+1}\right\|_{C}=0$.

\section{Numerical examples}

In this Section, the example of the previously considered ADS is investigated. We also construct the difference scheme with the Euler-Maclaurin formula instead of the trapezoidal rule and investigate numerically that the error of the method equals $O\left(\left(\frac{\tau}{m}\right)^{2 M+2}\right)$, where $m, M$ are the positive, natural numbers.

Let us consider the boundary-value problem

$$
\frac{\partial u}{\partial t}=\frac{\partial^{2} u}{\partial x^{2}}+u \ln u, 0<x<l, 0<t \leq T,
$$

where the boundary and initial conditions are coincidental with the exact solution $u(x, t)=$ $\exp \left\{B_{0} e^{t}+\frac{1}{2}-\frac{x^{2}}{4}\right\}$, and the function $Q(u)=u \ln u$ is a source for $u>1$ and a sink for $0<u<1$ [12]. We approximate it by the difference scheme

$$
\begin{gathered}
\frac{x_{i}^{n+1}-x_{i}^{n}}{\tau}=\left(\frac{1}{x_{i}^{n+1}-x_{i}^{n}} \int_{x_{i}^{n}}^{x_{i}^{n+1}} \frac{d x}{x^{2}-2}\right)^{-1}, x_{i}^{0} \in \bar{\omega}_{h}^{0}, i \in \mathbb{Z}, n=\overline{0, N_{0}-1}, \\
\frac{y_{i}^{n+1}-y_{i}^{n}}{\tau}=\varphi_{3}\left(y_{i}^{n}, y_{i}^{n+1}\right), y_{i}^{0}=u_{0}\left(x_{h i}^{0}\right), i \in \mathbb{Z}, n=\overline{0, N_{0}-1} .
\end{gathered}
$$

From (32) we find that $x_{i}^{n+1}=\sqrt{2+e^{\tau}\left(\left(x_{i}^{n}\right)^{2}-2\right)}$, where $0 \leq t_{n} \leq \ln \frac{2}{2-\left(x_{i}^{0}\right)^{2}}$ for $\left|x_{i}^{0}\right|<$ $\sqrt{2}$. To solve equation (33) we use iteration method (30). The stopping criterion in the iteration method is

$$
\left\|s^{s+1^{n+1}}-s^{n+1}\right\|_{\bar{C}_{n+1}} \leq \epsilon,
$$


Pobrane z czasopisma Annales AI- Informatica http://ai.annales.umcs.pl

Data: 26/04/2023 09:37:01

where $\epsilon$ is previously given tolerance. When the above condition is satisfied, we advance to the next level with $y_{i}^{n+1}={ }^{s+1^{n+1}}{ }_{i}$. Here $\left\|v^{n}\right\|_{\bar{C}_{n}}=\sup _{i \in P_{n}}\left|v_{i}^{n}\right|$, where $P^{n}$ determines the range of possible values for every index $i$ of the grid nodes on the $n-t h$ level.

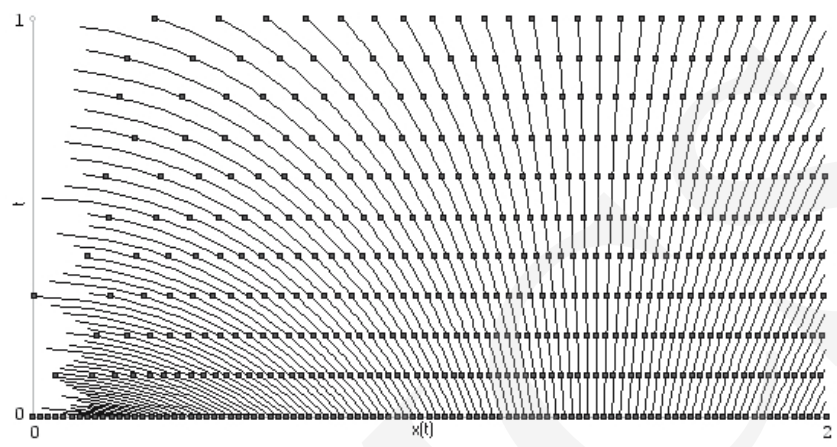

Fig. 1. The moving mesh of scheme (13)-(14).

Table 1 presents the results of the numerical experiments, where $S$ is the number of iterations.

Table 1. $l=2, T=1, \beta_{0}=0.5, \epsilon=1.0 \cdot 10^{-15}, h_{i}^{0}=0.02$.

\begin{tabular}{|c|c|c|c|c|}
\hline$\tau$ & $m$ & $\max _{0 \leq n \leq N_{0}}\left\|x_{h}^{n}-x\left(t_{n}\right)\right\|_{\bar{C}_{n}}$ & $\max _{0 \leq n \leq N_{0}}\left\|y^{n}-u_{h}\left(t_{n}\right)\right\|_{\bar{C}_{n}}$ & $S$ \\
\hline \hline 0.1 & 2 & $7.73 \cdot 10^{-18}$ & $2.01 \cdot 10^{-02}$ & 17 \\
& 10 & & $8.11 \cdot 10^{-04}$ & 17 \\
& 100 & & $8.11 \cdot 10^{-06}$ & 17 \\
& 1000 & & $8.11 \cdot 10^{-08}$ & 17 \\
\hline 0.01 & 2 & $1.60 \cdot 10^{-17}$ & $2.01 \cdot 10^{-04}$ & 8 \\
& 10 & & $8.04 \cdot 10^{-06}$ & 8 \\
& 100 & & $8.04 \cdot 10^{-08}$ & 8 \\
& 1000 & & $8.04 \cdot 10^{-10}$ & 8 \\
\hline
\end{tabular}

Now we investigate numerically stability of the considered scheme. We perturb the initial values $\tilde{y}_{i}^{0}=\tilde{u}_{0}\left(x_{i}^{0}\right)=u_{0}\left(x_{i}^{n}\right)+E_{S} \cos \left(100 x_{i}^{0} \pi / l\right), i \in P_{0}$ (see Fig. 2). 
Pobrane z czasopisma Annales AI- Informatica http://ai.annales.umcs.pl

Data: 26/04/2023 09:37:01

106 Difference schemes of arbitrary order of accuracy for semilinear parabolic equations

Table 2. $l=2, T=1, \beta_{0}=0.5, \epsilon=1.0 \cdot 10^{-15}, h_{i}^{0}=0.02, \tau=0.001, m=10$.

\begin{tabular}{|c|c|}
\hline$E_{S}$ & $\max _{0 \leq n \leq N_{0}}\left\|\tilde{y}^{n}-y\right\|_{\bar{C}_{n}}$ \\
\hline \hline $10^{0}$ & $1.23 \cdot 10^{+01}$ \\
$10^{-1}$ & $8.54 \cdot 10^{-01}$ \\
$10^{-2}$ & $8.20 \cdot 10^{-02}$ \\
$10^{-5}$ & $8.16 \cdot 10^{-05}$ \\
$10^{-10}$ & $8.16 \cdot 10^{-10}$ \\
\hline
\end{tabular}

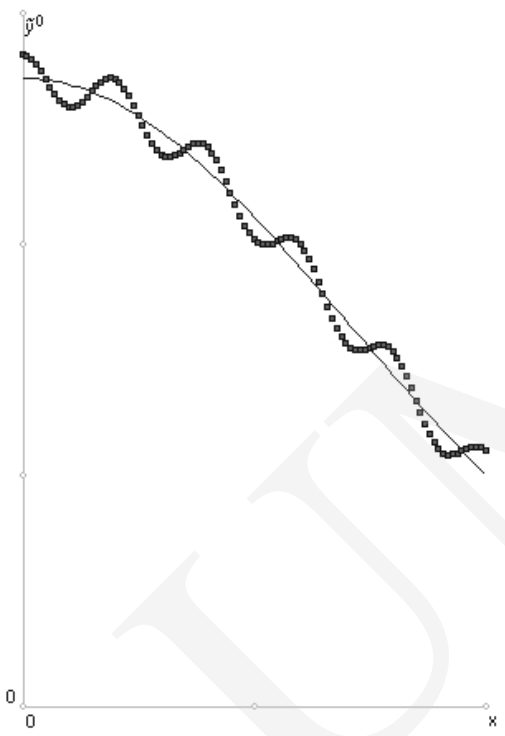

Fig. 2. The exact solution and $\tilde{y}_{i}^{0}$ for $l=2, T=1, \beta_{0}=0.5$, $E_{S}=10^{-1}, h_{i}^{0}=0.02$.

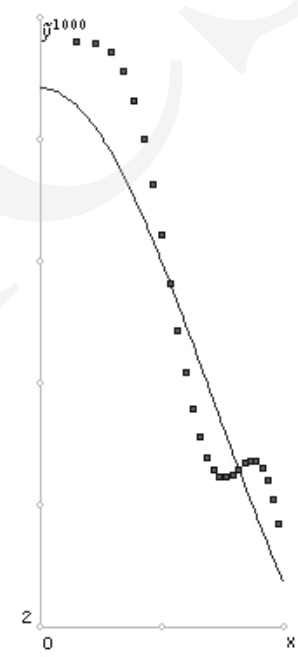

Fig. 3. The exact solution and $\tilde{y}_{i}^{1000}$ for $l=2, T=1, \beta_{0}=$ $0.5, E_{S}=10^{-1}, h_{i}^{0}=0.02$.

To obtain better numerical results, under the condition $f(u) \in C^{2 M+2}(\mathbb{R})$, where $M=$ const $>0$, we use the Euler-Maclaurin formula instead of the trapezoidal rule [2]

$$
\begin{gathered}
\int_{u_{i}^{n}}^{u_{i}^{n+1}} \frac{d x}{f(u)} \approx \frac{y_{i}^{n+1}-y_{i}^{n}}{m}\left(\frac{1}{2 f\left(y_{i}^{n+1}\right)}+\sum_{j=1}^{m-1} \frac{1}{f\left(y_{i}^{n}+j \frac{y_{i}^{n+1}-y_{i}^{n}}{m}\right)}+\frac{1}{2 f\left(y_{i}^{n}\right)}\right) \\
+\sum_{j=1}^{M}(-1)^{j} a_{j}\left(\frac{y_{i}^{n+1}-y_{i}^{n}}{m}\right)^{2 j}\left[\left(\frac{1}{f\left(y_{i}^{n+1}\right)}\right)^{(2 j-1)}-\left(\frac{1}{f\left(y_{i}^{n}\right)}\right)^{(2 j-1)}\right]
\end{gathered}
$$


where $a_{j}$ is calculated from $\frac{1}{2 M+1}=\frac{1}{2}+\sum_{j=1}^{M}(-1)^{j} \frac{(2 M) !}{(2 M-2 j+1) !} a_{j}$. We estimate a posteriori the error of this method [13]

$$
p^{N}=\log _{2}\left(D^{N} / D^{2 N}\right),
$$

where

$$
\begin{gathered}
D^{N}=\frac{1}{2^{2 M+2}-1} \max _{n=\overline{0, N_{0}}}\left\|y^{n}-y^{2 n}\right\|_{\bar{C}_{n}}, x_{i}^{n} \in \bar{\omega}, x_{i}^{2 n} \in \bar{\omega}_{h \tau / 2}, \\
D^{2 N}=\frac{1}{2^{2 M+2}-1} \max _{n=\overline{0,2 N_{0}}}\left\|y^{n}-y^{2 n}\right\|_{\bar{C}_{n}}, x_{i}^{n} \in \bar{\omega}_{h \tau / 2}, x_{i}^{2 n} \in \bar{\omega}_{h \tau / 4} .
\end{gathered}
$$

Here we use two additional grids $\bar{\omega}_{h \tau / 2}, \bar{\omega}_{h \tau / 4}$ with the time step twice and four times smaller than $\tau$, respectively. Tables $3-4$ present numerically that the error $\max _{0 \leq n \leq N_{0}}\left\|y^{n}-u^{n}\right\|_{\bar{C}_{n}}$ of the method applying the Euler-Macluarin formula equals $O\left(\left(\frac{\tau}{m}\right)^{2 M+2}\right)$.

Table 3. $\beta_{0}=0.5, l=2, T=1, M=1, \epsilon=1.0 \cdot 10^{-19}, h_{i}^{0}=0.2, m=1$.

\begin{tabular}{|l|c|c|c|}
\hline$\tau$ & $\max _{0 \leq n \leq N_{0}}\left\|y^{n}-u^{n}\right\|_{\bar{C}_{n}}$ & $D^{N}$ & $p^{N}$ \\
\hline \hline 0.5 & $6.67 \cdot 10^{-02}$ & - & - \\
\hline 0.25 & $3.43 \cdot 10^{-03}$ & $2.14 \cdot 10^{-04}$ & - \\
\hline 0.125 & $3.16 \cdot 10^{-04}$ & $1.98 \cdot 10^{-05}$ & 3.525 \\
\hline 0.0625 & $2.75 \cdot 10^{-05}$ & $1.72 \cdot 10^{-06}$ & 4.001 \\
\hline 0.03125 & $1.71 \cdot 10^{-06}$ & $1.07 \cdot 10^{-07}$ & 4.000 \\
\hline
\end{tabular}

Table 4. $\beta_{0}=0.5, l=2, T=1, M=2, \epsilon=1.0 \cdot 10^{-19}, h_{i}^{0}=0.2, m=1$.

\begin{tabular}{|l|c|c|c|}
\hline$\tau$ & $\max _{0 \leq n \leq N_{0}}\left\|y^{n}-u^{n}\right\|_{\bar{C}_{n}}$ & $D^{N}$ & $p^{N}$ \\
\hline \hline 0.5 & $2.80 \cdot 10^{-02}$ & - & - \\
\hline 0.25 & $4.32 \cdot 10^{-04}$ & $6.75 \cdot 10^{-06}$ & - \\
\hline 0.125 & $1.02 \cdot 10^{-05}$ & $1.59 \cdot 10^{-07}$ & 5.457 \\
\hline 0.0625 & $2.32 \cdot 10^{-07}$ & $6.32 \cdot 10^{-09}$ & 6.003 \\
\hline 0.03125 & $3.61 \cdot 10^{-09}$ & $5.65 \cdot 10^{-11}$ & 6.001 \\
\hline
\end{tabular}

\section{Conclusions}

In the paper, we have considered the EDS for the Cauchy problem for the semilinear parabolic equation. The solution with the separated variables $u(x, t)=X(x) T_{1}(t)+T_{2}(t)$ was a very important assumption. The ADSs have been constructed in the case when 
integrals in EDS cannot be evaluated exactly. The special attention was paid to investigate approximation, stability and convergence of the nonlinear scheme. Convergence of the iteration method was also considered. Numerical results have been presented to confirm the theoretical results given in the paper.

\section{References}

[1] Gavrilyuk I. P., Exact difference schemes and difference schemes of arbitrary given degree of accuracy for generalized one-dimensional third boundary value problem, $\mathrm{Z}$. Anal. Anwend. 12 (1993): 549-566.

[2] Kalitkin N. N., The Euler-MacLaurin formula of hight orders (in Russian), Mathematical Modelling 16(10) (2004): 64-66.

[3] Lapinska-Chrzczonowicz M., Matus P., Exact difference schemes for parabolic equations, International Journal of Numerical Analysis and Modeling 5(2) (2008): 303 319.

[4] Lapinska-Chrzczonowicz M., Exact difference schemes for multidimensional heat conduction equations, Computational Methods In Applied Mathematics 7(2) (2007): 178-190.

[5] Lapinska-Chrzczonowicz M., Exact difference schemes and difference schemes of arbitrary order of approximation for multidimensional heat conduction equations, Proc. of the Third International Conference Computational Methods In Applied Mathematics CMAM-3 (Minsk, 2007): 106-121.

[6] Makarov V. L., Gavrilyuk I. P., Kutniv M. V., Hermann M., A two-point difference scheme of an arbitrary order of accuracy for BVPs for system of first order nonlinear ODEs, Computational Methods In Applied Mathematics 4(4) (2004): 464-483.

[7] Matus P., Irkhin U., Lapinska-Chrzczonowicz M., Exact difference schemes for timedependent problems, Computational Methods In Applied Mathematics 5(4) (2005): 422 448.

[8] Matus P., Irkhin U., Lapinska-Chrzczonowicz M., Lemeshevsky S.V., On exact finitedifference schemes for hyperbolic and parabolic equations, Differentsial'nye Uravneniya 43(7) (2007): 978-986.

[9] Mickens R.E. Applications of Nonstandard Finite Difference Schemes (World Scientific Publishing, Singapore, 2000).

[10] Paradzinska A., Matus P., High accuracy difference schemes for nonlinear transfer equation $\frac{\partial u}{\partial t}+u \frac{\partial u}{\partial x}=f(u)$, Mathematical Modelling and Analysis 12(4) (2007): 469482.

[11] Samarskii A. A., The Theory of Difference Schemes (Marcel Dekker Inc., New YorkBasel, 2001).

[12] Samarskii A. A., Galaktinov V. A., Kurdymov S. P., Mikhailov A. P., Blow-up in Quasilinear Parabolic Equations (Walter de Gruyter, Berlin, New York, 1995).

[13] Smarskii A. A., Gulin A. W., Numerical Methods (in Russian) (Nauka, Moscow, 1989). 
Pobrane z czasopisma Annales AI- Informatica http://ai.annales.umcs.pl

Data: 26/04/2023 09:37:01

[14] Stoer J., Bulirsch R., Introduction to Numerical Analysis (in Polish) (PWN, Warsaw, 1987). 